\title{
Approximate Treatment of Lepton Distortion in Charged-Current Neutrino Scattering from Nuclei
}

\author{
Jonathan Engel \\ Department of Physics and Astronomy, University of North Carolina, Chapel Hill, NC 27599-3255
}

March 13, 2018

\begin{abstract}
The partial-wave expansion used to treat the distortion of scattered electrons by the nuclear Coulomb field is simpler and considerably less timeconsuming when applied to the production of muons and electrons by low and intermediate-energy neutrinos. For angle-integrated cross sections, however, a modification of the "effective-momentum-transfer" approximation seems to work so well that for muons the full distorted-wave treatment is usually unnecessary, even at kinetic energies as low as an MeV and in nuclei as heavy as lead. The method does not work as well for electron production at low energies, but there a Fermi function usually often proves perfectly adequate. Scattering of electron-neutrinos from muon decay on iodine and of atmospheric neutrinos on iron are discussed in light of these results.
\end{abstract}




\section{Introduction}

Converting neutrinos with energies below a few hundred $\mathrm{MeV}$ into electrons or muons by scattering them from nuclei is useful in the search for new physics. Radiochemical detectors [1] rely on neutrino-nucleus scattering to measure the apparently too low solar neutrino flux. Proton-decay experiments, doubling as neutrino observatories, use neutrino scattering from oxygen or iron to look for oscillations of neutrinos produced in the atmosphere 2, 3]. And experiments at Los Alamos [4] measure neutrino-carbon cross sections, in part to check the flux in a beam that has reportedly produced neutrino oscillations in the lab.

Because neutrinos interact so weakly the Born approximation to neutrino-nucleus scattering should be accurate. The only difficulty with this first-order plane-wave approach is that the electrons or muons produced when the neutrinos interact feel an electrostatic force from the nucleus as they leave. In heavy nuclei the Coulomb force can have large effects and even in light nuclei it changes cross sections noticeably. The best way to include the Coulomb interaction is through the distorted-wave Born approximation (DWBA) [5], which entails an expansion of the outgoing wave function in partial waves. In processes similar to neutrino scattering but more often measured, two approximations help avoid the cumbersome and time-consuming partial-wave expansion. Calculations of beta decay, which produces low-energy electrons, contain a Fermi function that multiplies the decay amplitude by the ratio of Coulomb to free Dirac s-wave functions at the nuclear radius [6]. In electron scattering, at energies much larger than the electrostatic potential energy in the nucleus, an "effective momentum approximation" (EMA) uses plane waves with shortened wavelengths and increased amplitudes in place of incoming and outgoing distorted waves [7]. Both approximations are substantial improvements over the unrenormalized plane-wave impulse approximation, though the EMA predictions for differential cross sections still differ significantly from those of the full DWBA in heavy nuclei, even at high energies [8].

This paper discusses the application of these very convenient approximations and modifications thereof to charged-current neutrino-nucleus scattering, for which the outgoing lepton is either an electron or muon. Because the neutrino is not charged and the weak interaction is short-ranged, the expansion in partial waves is less complicated and timeconsuming than in electron scattering. But thus far the most significant observable in all the neutrino experiments cited above is the total cross section, and one might question whether the full DWBA is really required to obtain it. The two approximations just discussed preserve the intuitive plane-wave picture of the scattering and are so much easier to apply that one really ought to retain them if possible. On the other hand, it is not clear that they will always work well; the EMA, for example, has been derived only for extremely relativistic particles [9, 10, 11], while the most energetic outgoing muons considered here are far from the relativistic limit. I show below however that although the Fermi function is accurate only for low-energy electrons in most nuclei, a simple modification allows the EMA to work remarkably well (better than in electron scattering), even in heavy nuclei and, for muons, even at very low energies. 


\section{General Considerations}

The differential cross section for a neutrino of momentum $\vec{k}_{\nu}$ to produce a muon or electron at scattered angle $\Omega$ and energy $E$, with the nucleus going from state $|i\rangle$ to state $|f\rangle$, can be written as a phase space factor times a sum of squared matrix elements, viz:

$$
\begin{array}{r}
\frac{\partial^{2} \sigma}{\partial E \partial \Omega}=\frac{G^{2} k E}{4 \pi^{2}} \sum_{s}|\mathcal{M}(i \rightarrow f)|^{2} \\
\mathcal{M}_{s}(i \rightarrow f)=\int d^{3} r \bar{\psi}_{\vec{k}, s}^{-}(\vec{r}) \gamma_{\mu} \nu_{\vec{k}_{\nu}} e^{i \overrightarrow{k_{\nu}} \cdot \vec{r}}\left\langle f\left|\mathcal{J}^{\mu}(\vec{r})\right| i\right\rangle,
\end{array}
$$

where $G$ is the Fermi coupling constant, $\mathcal{J}$ is the charged weak nuclear current, $\nu_{\vec{k}_{\nu}}$ is the 4-spinor containing the momentum-space components of the left-handed neutrino plane wave with momentum $\vec{k}_{\nu}$, and $\psi_{\vec{k}, s}^{-}(\vec{r})$ is the charged lepton (electron or muon) wave function with spin projection $s$ and energy $E=\sqrt{k^{2}+M^{2}}$ in the electrostatic potential

$$
\begin{array}{ccc}
V= & \frac{-Z \alpha}{2 R}\left(3-r^{2} / R^{2}\right) & r<R \\
V= & -Z \alpha / r & r>R
\end{array} .
$$

( $R$ is the nuclear radius.) The wave function $\psi^{-}$is an eigenstate of the full Hamiltonian, electrostatic potential included, that looks asymptotically like a plane plus an ingoing spherical wave. Expanded in partial waves, it has the form 12, 13]

$$
\psi_{\vec{k}, s}^{-}=\frac{1}{\sqrt{\pi} k} \sqrt{\frac{E+M}{E}} \sum_{l, j, m} i^{l}\left\langle l m-s, \frac{1}{2} s \mid j m\right\rangle Y_{l m}^{*}(\hat{r}) e^{-i \delta_{j, l}} \frac{1}{r}\left(\begin{array}{c}
i P_{j, l}^{E}(r) \Omega_{l, j m}(\hat{r}) \\
Q_{j, l}^{E}(r) \Omega_{2 j-l, j m}(\hat{r})
\end{array}\right)
$$

where the quantum number $j$ and the label $l$ determine the the eigenvalue $\kappa=(l-$ $j)(2 j+1)$ of the operator $K=-\beta(2 \vec{L} \cdot \vec{S}+1)$ ( $\beta$ is the usual Dirac-equation matrix); $\Omega_{l, j m}(\hat{r}) \equiv\left[Y_{l} \chi\right]_{m}^{j}$ is a two-spinor with non-relativistic quantum numbers $l, s=\frac{1}{2}$, and $j$; and $\delta_{j, l}$ is the "inner phase shift" [13]. The radial wave functions $P_{j, l}^{E}(r)$ and $Q_{j, l}^{E}(r)$ obey the equations

$$
\begin{aligned}
\frac{d P_{j, l}^{E}(r)}{d r} & =-\frac{\kappa}{r} P_{j, l}^{E}(r)-[E+M-V(r)] Q_{j, l}^{E}(r) \\
\frac{d Q_{j, l}^{E}(r)}{d r} & =\frac{\kappa}{r} Q_{j, l}^{E}(r)-[E-M-V(r)] P_{j, l}^{E}(r) .
\end{aligned}
$$

Since the goal here is to test approximations that preserve the easily interpreted planewave formalism, I will use only the vector charge and not the full current in Eq. (2); the charge, for which full distorted-wave cross-section formulae can be displayed and calculated simply, should be sufficient. If the nuclear target states have SU(4) symmetry the major part of the axial-vector current will affect the matrix element in essentially the same way

\footnotetext{
${ }^{1}$ The total angular momentum $j$ is a good quantum number but the orbital angular momentum $l$ is not and serves as a label here.
} 
as the charge. There is no obvious reason to think that the rest of the current will change the results in a qualitative way.

Using the upper and lower radial wave functions $P_{j l}^{E}(r)$ and $Q_{j l}^{E}(r)$ from Eq. (5), one can define

$$
\begin{aligned}
F_{L J, j}^{ \pm} & =\int d^{3} r \frac{1}{r} P_{j, j \pm \frac{1}{2}}^{E}(r) j_{L}\left(k_{\nu} r\right) Y_{J 0}(\hat{r}) \rho_{f i}(\vec{r}) \\
G_{L J, j}^{ \pm} & =\int d^{3} r \frac{1}{r} Q_{j, j \pm \frac{1}{2}}^{E}(r) j_{L}\left(k_{\nu} r\right) Y_{J 0}(\hat{r}) \rho_{f i}(\vec{r})
\end{aligned}
$$

where

$$
\rho_{f i}(\vec{r})=\left\langle f\left|\sum_{n} \delta\left(\vec{r}-\overrightarrow{r_{n}}\right) \tau_{n}^{+}\right| i\right\rangle
$$

is the isovector transition transition density and the sum is over nucleons $n$. The angleintegrated cross section, which is of particular interest, takes a relatively simple form if the recoil of the nucleus is neglected, since $k$ and $E$ are then held fixed while $\Omega$ is integrated over, with the result that the cross section does not depend directly on the phase shifts and the partial waves do not interfere with one another. The general expression for a final state with arbitrary $J^{\pi}$ (and initial state with $0^{+}$), restricting the current to the vector charge, is

$$
\sum_{j, L}\left(\begin{array}{c}
\frac{d \sigma}{d E}=G^{2} \frac{E+m}{2 k} \times \\
-2\left\langle j+\frac{1}{2} 0, L 0 \mid J 0\right\rangle \\
j^{2} \hat{L}^{2}\left[\left\langle j-\frac{1}{2} 0, L 0 \mid J 0\right\rangle^{2}\left(F_{L J, j}^{-}{ }^{2}+G_{L J, j}^{+}{ }^{2}\right)+\left\langle j+\frac{1}{2} 0, L 0 \mid J 0\right\rangle^{2}\left(F_{L J, j}^{+}{ }^{2}+G_{L J, j}^{-}{ }^{2}\right)\right] \\
\left.\begin{array}{l}
\sqrt{\left[J^{2}-\left(L+\frac{1}{2}-j\right)^{2}\right]\left[(J+1)^{2}-\left(L+\frac{1}{2}-j\right)^{2}\right]} \\
\left\langle j-\frac{1}{2} 0, L+10 \mid J 0\right\rangle\left(F_{L+1 J, j}^{-} G_{L J, j}^{-}-F_{L J, j}^{+} G_{L+1 J, j}^{+}\right) \\
+\sqrt{\left[J^{2}-\left(L+\frac{1}{2}+j\right)^{2}\right]\left[(J+1)^{2}-\left(L+\frac{1}{2}+j\right)^{2}\right]} \\
\left\langle j-\frac{1}{2} 0, L-10 \mid J 0\right\rangle\left(F_{L-1 J, j}^{-} G_{L J, j}^{-}-F_{L J, j}^{+} G_{L-1 J, j}^{+}\right)
\end{array}\right]
\end{array}\right) .
$$

This expression will be used below for comparing exact results with approximations. I will take the density in Eq. (7) to have the form $\rho_{f i} \propto \delta(r-R) Y_{J M}(\hat{r})$ for transitions from a $0^{+}$ground state to excited states with angular momentum $J$ and projection $M$, so that, e.g., $1^{-}$states correspond to the isobar analogs of Goldhaber-Teller collectivedipole excitations. There are several reasons for this choice: densities for most low-lying collective states are surface-peaked, placing the strength at the nuclear radius provides a severe test for the approximations below (which work best at small $r$ ) and, finally, densities for higher-energy noncollective states can be represented as superposed densities peaked at different points in the nucleus, so that a $\delta$-function density should be sufficiently general.

\section{The Fermi Function}

It is convenient to think about the effects of an electrostatic potential on an outgoing particle in terms of a local effective energy and momentum inside the nucleus:

$$
E_{\text {eff }}=E-V(0), \quad k_{\text {eff }}=\sqrt{E_{\text {eff }}^{2}-M^{2}}
$$


When $k_{\text {eff }} R<<1$, a Fermi function of $Z$ and $E$ that multiplies the outgoing wave is appropriate. In this low "effective-momentum" limit, only s-waves contribute to the scattering, and they vary nearly linearly inside the nucleus; the Fermi function can then be taken to be the ratio of, e.g., the Coulomb wave to the free wave at the nuclear surface, a quantity given by

$$
F(Z, E)=2\left(1+\gamma_{0}\right)(2 k R)^{-2\left(1-\gamma_{0}\right)} e^{\pi \nu} \frac{\left|\Gamma\left(\gamma_{0}+i \nu\right)\right|^{2}}{\Gamma\left(2 \gamma_{0}+1\right)^{2}} \quad, \quad \gamma_{0}=\sqrt{1-Z^{2} \alpha^{2}} \quad, \quad \nu= \pm \frac{Z \alpha E}{k} .
$$

The Fermi function is useful for low-energy electrons and is often employed in calculations of beta decay, but is not likely to work well for muons except in light nuclei. The reason is that for nonrelativistic muons, $k_{\text {eff }} \approx \sqrt{2 M_{\mu} E_{\text {eff }}} \geq \sqrt{2 M_{\mu} V_{0}}$, which even in ${ }^{12} \mathrm{~N}$, the product of a charge-exchange reaction on ${ }^{12} \mathrm{C}$, implies that $k_{\mathrm{eff}} R \lesssim .5$ (large enough to cause a $10 \%$ error with the Fermi function above). Thus, even when the energy at infinity is so low that only s-wave muons are produced the usual Fermi function will generally not be not very accurate. Figure 11 shows the total cross section for exciting a (fictitious) $0^{+}$ state in ${ }^{208} \mathrm{Bi}$ at $15 \mathrm{MeV}$ with a surface-peaked transition density, as a function of outgoing lepton kinetic energy for both electrons and muons; the $0^{+}$multipole is the one with the largest s-wave contribution at low energies so the choice should maximize the accuracy of the Fermi function. Indeed, for electrons, the function does well for the lowest $5-10 \mathrm{MeV}$ of electron energy. For muons, however, the approximation is never valid. Its accuracy can be improved significantly by using the s-wave solution from the potential in Eq. (3), rather than the pure Coulomb potential (see Ref. [6]), but except at low energies where such functions are tabulated, that means solving the s-wave Dirac equation, a task that is simpler than carrying out the full DWBA but still relatively involved.

One physical situation in which these issues are important is the scattering of electron neutrinos produced by muon-decay. A few years ago an attempt was made to calibrate an ${ }^{127}$ I solar-neutrino detector by exposing it to a flux of muon-decay neutrinos at LAMPF 14]. The neutrinos have much higher energy than their solar counterparts and excite the nucleus in different ways, so that a good understanding of low-lying states in ${ }^{127} \mathrm{Xe}$ is required to extract the solar-neutrino cross section. In addition, the outgoing electron often has too high an energy to allow use of the usual Fermi function (but not high enough for the EMA); if the transition density is surface-peaked and concentrated at an average of $6 \mathrm{MeV}$, the Fermi function gives values about $50 \%$ larger than the DWBA $0^{+}$multipole cross section when only the vector charge is included. The difficulties with nuclear structure and electron distortion were discussed in ref. [15]; to treat the distortion for the $0^{+}$and $1^{+}$components of the cross section, the authors relied on a fit 16] to tables in Ref. [6], which use the potential in Eq. (3) rather than the pure Coulomb force to calculate the Fermi function. Here that procedure still misses the cross sections, though only by about 10 or $15 \%$. The difference is due mainly to higher partial waves, which are affected differently by the electrostatic force than the s-wave that underlies the Fermi function. It would take a significantly larger difference, however, to alter the conclusion of Ref. [15] that the LAMPF cross sections are too large to be understood or interpreted. 
Figure 1: The total cross section for scattering from the ground states of ${ }^{12} \mathrm{C}$ and ${ }^{208} \mathrm{~Pb}$ to (fictitious) $0^{+}$states at $15 \mathrm{MeV}$ in ${ }^{12} \mathrm{~N}$ and ${ }^{208} \mathrm{Bi}$, with transition densities proportional to $\delta(r-R)$, as a function of outgoing lepton kinetic energy for both electrons (a) and muons (b). The solid lines represent the full DWBA results, the dotted lines neglect the Coulomb force completely, and the dashed lines are the Fermi-function approximations.

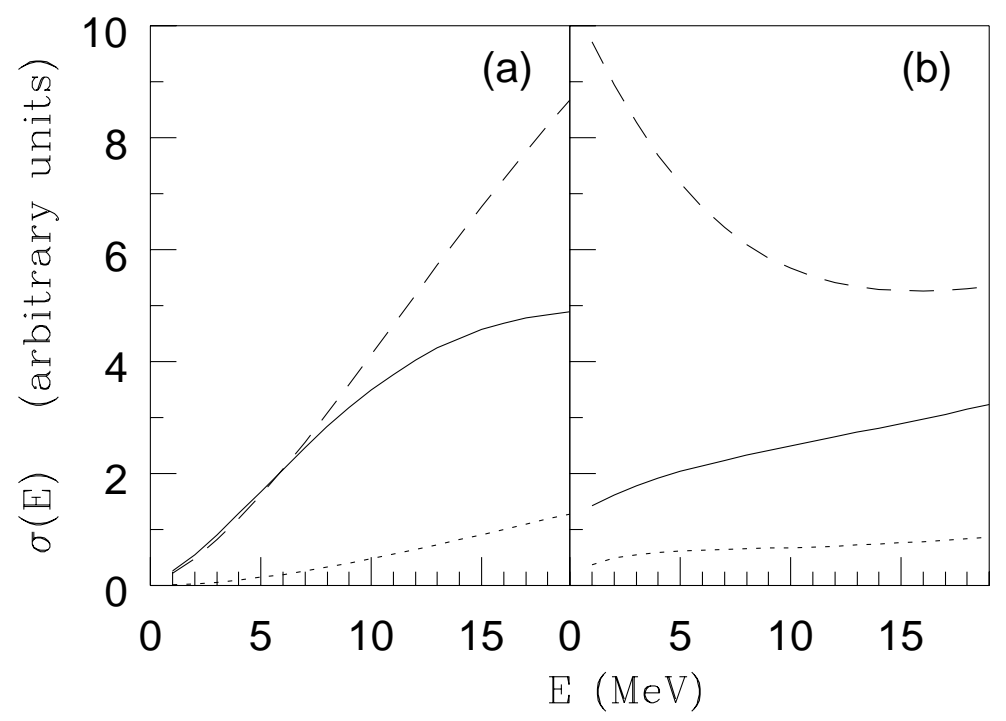

\section{The EMA and a Modified EMA}

At higher energies, the Fermi function doesn't work even for electrons and we need a different approximation. In electron scattering, the EMA is used to untangle the electrostatic attraction of the electron to the nucleus from the single hard-photon exchange. As already mentioned, in its simplest form this approximation consists of shortening the electron wavelength $k$ to $k_{\text {eff }}$ inside the nucleus, resulting in a larger effective momentum transfer, and rescaling the amplitude of the wave function by $k_{\text {eff }} / k$. The procedure's accuracy depends on scattering angle and decreases significantly as $Z$ grows; it is not regarded as good in lead. But the application of the EMA to electrons produced by neutrino scattering, which is straightforward, should work better than its application in electron scattering. One reason is that only the outgoing wave experiences a Coulomb force; a more significant one is that the range of the weak interaction is short. In electron scattering regions far from the nucleus, where the local momentum is quite different from $k_{\text {eff }} / k$, affect the scattering matrix element because of long-range photon exchange. Here by contrast the only effects are from inside the nucleus. Figure 2 shows the total cross section for scattering from the ground states of ${ }^{12} \mathrm{C}$ and ${ }^{208} \mathrm{~Pb}$ to Goldhaber-Teller (ana$\log$ ) resonances in ${ }^{12} \mathrm{~N}$ and ${ }^{208} \mathrm{Bi}$, along with the EMA predictions. The approximation is quite good above about $30 \mathrm{MeV}$ in lead, leaving only a small kinematic window in which neither the EMA nor a Fermi function works. For electrons, therefore, one can accurately account for the effects of the electrostatic potential without using the DWBA except in 
restricted kinematic regions in heavy nuclei.

Figure 2: The total cross section for scattering from the ground states of ${ }^{12} \mathrm{C}$ (a) and ${ }^{208} \mathrm{~Pb}$ (b) to (fictitious) $0^{+}$states at $15 \mathrm{MeV}$ in ${ }^{12} \mathrm{~N}$ and ${ }^{208} \mathrm{Bi}$, as a function of outgoing kinetic energy and with transition densities proportional to $\delta(r-R)$. The solid lines are the full DWBA results, the dotted lines neglect the Coulomb force completely, and the dashed lines are the EMA results.

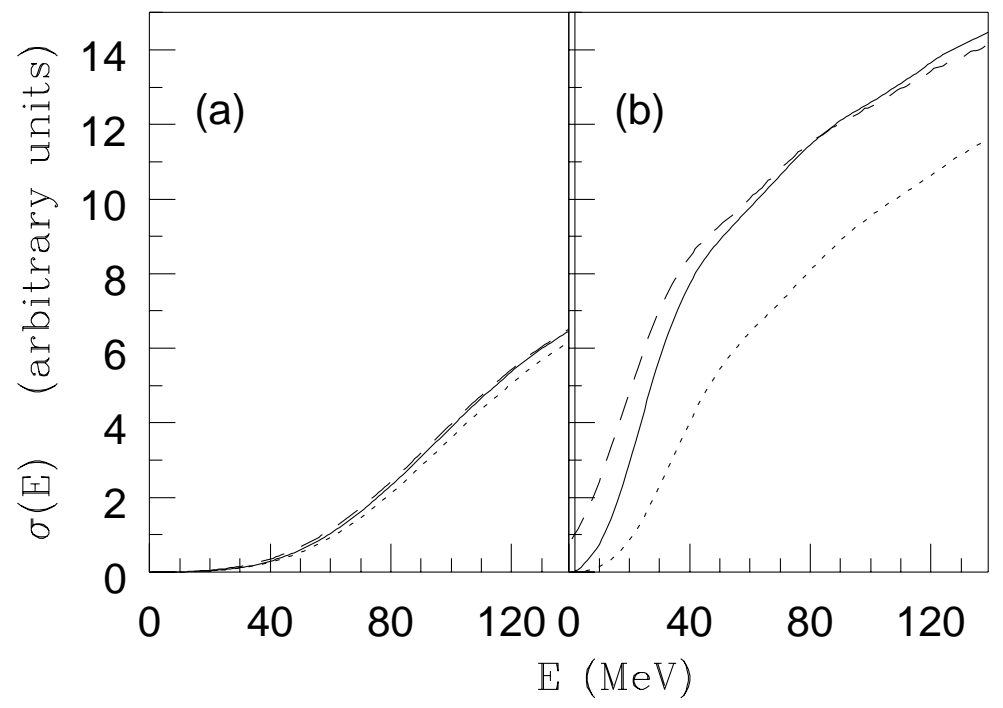

Muons are another story however; the best way to extend the EMA to massive particles is not immediately clear. The approximation and corrections to it have been derived from the high-energy limit of the Dirac equation, either through an eikonal approximation 9 or an expansion in inverse powers of $k[10]$. Both approaches begin by neglecting the mass of the electron and so separating the usually coupled left and right handed spinors, hence simplifying the equation. Here we are concerned with muon energies below a $\mathrm{GeV}$, for which the rest mass is not negligible. The basic ideas behind the simplest approximation that the Coulomb potential shortens the wavelength in the interaction region and increases the wave amplitude by focusing or defocusing the particles as they approach or leave - do not appear to hinge on the particles' rest mass, as long as the wavelength is short enough for a local momentum to have some meaning. Some version of the EMA should therefore apply to intermediate-energy muons as well as electrons.

The shortening of the wavelength will clearly work the same way for the two kinds of particle, the only difference being the kinematic relation between $k_{\text {eff }}$ and $E_{\text {eff }}$, but what about the amplitude rescaling? Should it still be $k_{\text {eff }} / k$ or some other factor that involves the muon mass? We can decide without extending the the full analysis of Refs. [9, 10] by viewing the change in amplitude as follows: The use of a plane-wave approximation in the interaction region is equivalent to the assumption that the Coulomb potential does not cause the particles to alter their direction very much when they approach or leave the nucleus. It should therefore not strongly alter an outgoing muon wave packet, which 
asymptotically is spherical, after it leaves the nucleus except by slowing it down and thereby changing the average radial wavelength and amplitude as the wave moves to larger $r$. (In particular, it should not cause much reflection.) But the differential cross section is directly related to the radial integral of the square of the wave function over all $r$ at a fixed solid angle (in the large time limit), and under the assumptions above the Coulomb interaction should not affect this quantity much once the outgoing wave moves beyond the nucleus. Since most of the effect it does have will be to redistribute flux from one angle to another, the total cross section should be nearly the same as if the wave kept the same radial wavelength it had inside the nucleus, i.e. as if the potential were equal to $V(0)$ everywhere in space. For that situation the plane-wave approximation is exact to lowest order in $Z \alpha$ if the effective momentum and energy $k_{\text {eff }}$ and $E_{\text {eff }}$ replace the real quantities $k$ and $E$ everywhere in Eq. (2). In other words, besides shortening the wavelength in the matrix element $\mathcal{M}$, one should replace the phase space factor $k E$ by $k_{\text {eff }} E_{\text {eff }}$ 17]. In the Born approximation this is equivalent to a change in the muon wave function

$$
e^{i \vec{k} \cdot \vec{r}} \longrightarrow \sqrt{\frac{k_{\mathrm{eff}} E_{\mathrm{eff}}}{k E}} e^{i \vec{k}_{\mathrm{eff}} \cdot \vec{r}}
$$

i.e. a rescaling of the amplitude by $\sqrt{k_{\text {eff }} E_{\text {eff }} / k E}$ rather than $k_{\text {eff }} / k$. I will call this approximation the "modified EMA".

With this alteration the EMA in fact generally works better for muons than for electrons at low energies for the same reason the Fermi function doesn't work as well: the muon mass guarantees that $k_{\text {eff }} R$ never drops below $\sqrt{2 M_{\mu} V(0)} R \approx 0.5$ in nitrogen and 2.6 in bismuth. Figure 3 shows total cross sections for exciting several states with different angular momenta in $\mathrm{Bi}$ (again assuming a surface peaked transition density) alongside the modified EMA predictions and those of the usual EMA, in which the plane wave is scaled by $k_{\text {eff }} / k$. The usual EMA is pathological at low energies, but the modified EMA agrees extremely well with the exact solutions except for the $0^{+}$multipole, where it is off by a little over $5 \%$ at muon energies of $150 \mathrm{MeV}$. (There are fewer ways of coupling neutrino and muon angular momenta to $0^{+}$, which may accentuate errors in any one partial wave for that multipole.) The differences in lighter nuclei are less; in ${ }^{56} \mathrm{Fe}$ the agreement is almost exact for all multipoles except at very low energies, where the $0^{+}$multipole still is somewhat worse than the others. One might see similar behavior in the $1^{+}$multipole, also "allowed", were the entire current included. But the modified EMA is still dramatically better than the usual plane-wave treatment, even for $0^{+}$transitions in heavy nuclei.

Thus far the only significant laboratory experiments with muon neutrinos in this energy range are the LAMPF measurements of the cross section for neutrinos produced by the decay of pions in flight scattering from ${ }^{12} \mathrm{C}$. Because $Z$ is small, electrostatic effects are likewise small. Most of the difference between the DWBA and plane-wave approximations in this light nucleus was correctly accounted for in Ref. [18], which rescaled the muon phase space as prescribed by the modified EMA without altering the nuclear matrix element (though the definition of $k_{\text {eff }}$ is reported incorrectly in the manuscript). Another situation worth examining, however, is the scattering of atmospheric muon and electron neutrinos from the iron in the SOUDAN proton-decay detector. Results of experiments there and 
Figure 3: Total cross sections for several multipole excitations (labeling the panels) from the ground state of ${ }^{208} \mathrm{~Pb}$ to fictitious states at $15 \mathrm{MeV}$ in ${ }^{208} \mathrm{Bi}$, as a function of outgoing kinetic energy and with transition densities proportional to $\delta(r-R) Y_{J M}$. The solid lines are the results of full DWBA, the dotted lines those with the Coulomb force neglected, the dashed lines those of the usual EMA used in electron scattering, and the dot-dashed lines those of the modified EMA.

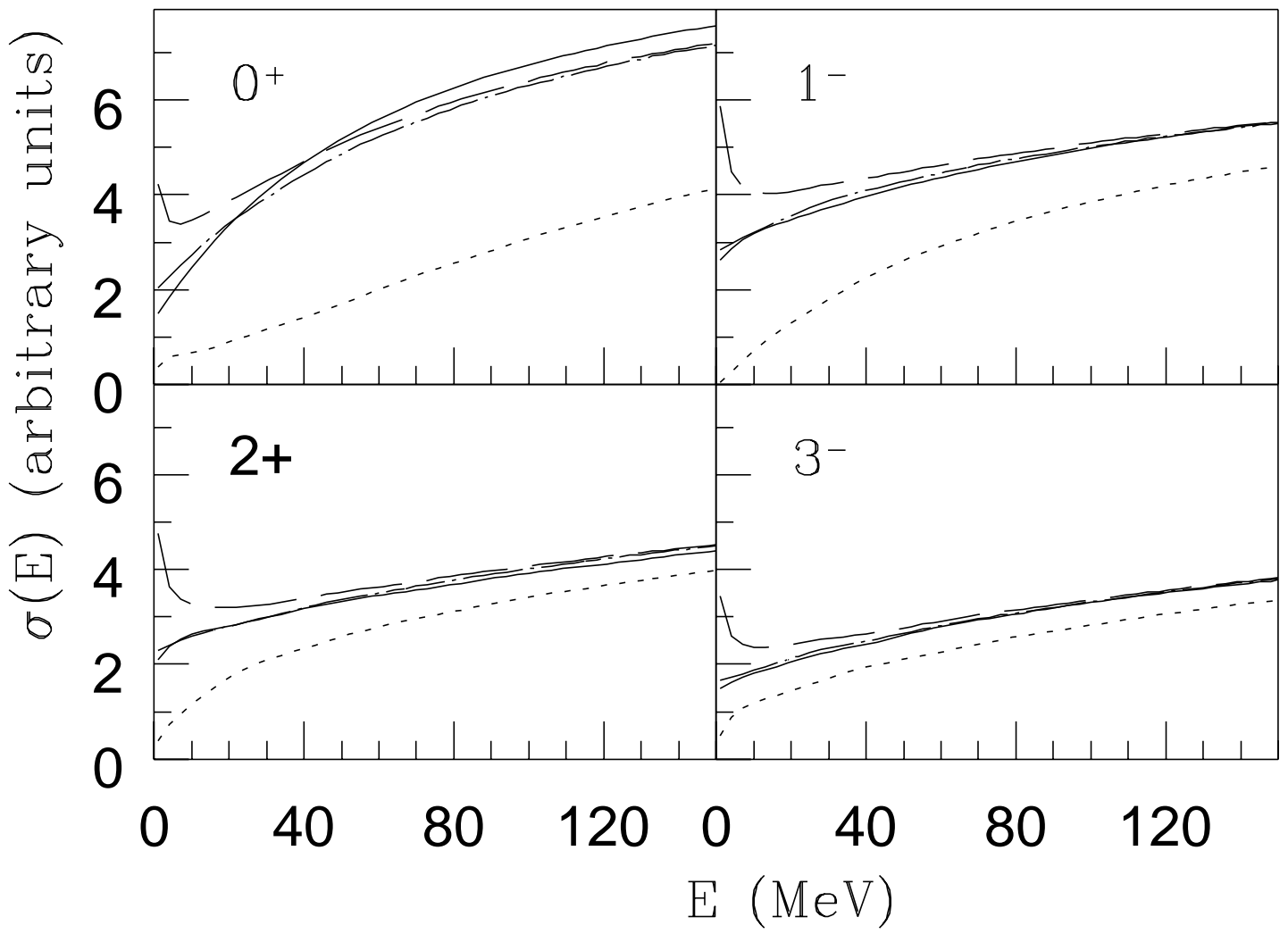

at other places imply that the ratio of muon-neutrino flux to electron-neutrino flux in the atmosphere is about $50-80 \%$ of the expected ratio[3]. The analyses use the Fermi-gas model of the nucleus and don't generally account for final-state effects in quasielastic scattering. Table 1 shows the changes due to the electrostatic force (calculated in the modified EMA) from the simple Fermi-gas model predictions for the quasielastic production of muons, antimuons, electrons, and positrons in iron. I have chosen relatively low outgoing momenta and taken neutrino fluxes from Ref. [19]. The individual event rates actually change significantly for these momenta, but as is usually the case, the ratio of total $\mu$ to total $e$ events hardly budges, so that one one apparently can safely neglect Coulomb effects even in a material as heavy as iron. 
Table 1: Calculated event rates for atmospheric neutrinos in ${ }^{54} \mathrm{Fe}$, in arbitrary units, with and without Coulomb distortion of the final-state leptons.

\begin{tabular}{lccc}
\hline \hline & $100 \mathrm{MeV}$ & $200 \mathrm{MeV}$ & $300 \mathrm{MeV}$ \\
\hline$\nu_{\mu^{-}}($no Coul. $)$ & 231 & 506 & 467 \\
$\nu_{\mu^{-}}$ & 165 & 453 & 446 \\
$\nu_{e^{-}}$ & 134 & 294 & 258 \\
$\nu_{e^{-}}($no Coul. $)$ & 104 & 265 & 247 \\
$\nu_{\mu^{+}}($no Coul. $)$ & 28 & 64 & 69 \\
$\nu_{\mu^{+}}$ & 40 & 70 & 72 \\
$\nu_{e^{+}}($no Coul. $)$ & 24 & 38 & 38 \\
$\nu_{e^{+}}$ & 29 & 41 & 39 \\
$\left(\nu_{\mu^{-}}+\nu_{\mu^{+}}\right) /\left(\nu_{e^{-}}+\nu_{e^{+}}\right)$ & 1.65 & 1.72 & 1.81 \\
$\left(\nu_{\mu^{-}}+\nu_{\mu^{+}}\right) /\left(\nu_{e^{-}}+\nu_{e^{+}}\right)($no Coul. $)$ & 1.54 & 1.70 & 1.81 \\
\hline
\end{tabular}

\section{Conclusions}

The chief result of this paper is that the effective-momentum approximation works better in neutrino scattering than in electron scattering, particularly for muons, where the approach has to be modified slightly to take into account the charged particle's mass. It probably doesn't work quite as well for differential cross sections but preliminary analysis (on $0^{+}$transitions with the vector charge) indicate that the modified EMA is still surprisingly good. The real scattering redistributes some flux from forward angles to places where the cross section is lower, but not as much as one might imagine. In any event, we are not likely to pay attention to details of weak-interaction differential cross sections in the near future. For total cross sections the modified EMA works admirably for muons down to low energies and the usual EMA just as well for electrons except in a region where a Fermi function is often adequate. In heavy nuclei there is a kinematic window for electrons in which neither approximation is completely sufficient, but it is small. Important examples from this window - neutrinos from muon decay on iodine is one - are not easy to find at present.

This work was supported by the U.S. Department of Energy under grant DE-FG0297ER41019. I thank E. Kolbe and P. Vogel for useful discussions and correspondence. 


\section{References}

[1] J.N. Bahcall, Neutrino Astrophysics (Cambridge University Press, Cambridge, England, 1989).

[2] K. S. Hirata et al., Phys. Lett. B205, 416 (1988); Phys. Lett. B280, 146 (1992)

[3] W.W.M., Allison et. al., Phys. Lett. B391, 491 (1997).

[4] C. Athanassopoulos et al., Phys. Rev. Lett. 77, 3082 (1996); C. Athanassopoulos et al., Phys. Rev. C54, 2685 (1996).

[5] See, e.g., G. Cò and J. Heisenberg, Phys. Lett. B197, 489 (1987).

[6] H. Behrens and W. Bühring, Electron Radial Wave Functions and Nuclear Beta-decay (Clarendon Press, Oxford, England, 1982).

[7] M. Traini, S. Turck-Chièze, and A. Zghiche, Phys. Rev. C38, 2799 (1988); C. Giusti and F.D. Pacati, Nucl. Phys. A473, 717 (1987); C. Giusti and F.D. Pacati, Nucl. Phys. A485, 461 (1987).

[8] K.S. Kim, L.E. Wright, Yanhe Jin; D.W. Kosik, Phys. Rev. C54, 2515 (1996).

[9] D.R. Yennie, F.L. Boos, and D.G. Ravenhall, Phys. Rev. 137, B882 (1965).

[10] F. Lenz and R Rosenfelder, Nucl. Phys. A176, 513 (1971) and references therein.

[11] J. Knoll, Nucl. Phys. A223, 462 (1974).

[12] A.I. Akhiezer and V.B. Beresteckii,Quantum Electrodynamics (John Wiley and Sons, New York, 1965).

[13] F. Salat, J.M. Fernández-Varea, and W. Williamson, Jr., Comp. Phys Comm. 62, 65 (1991).

[14] B.T. Cleveland, T. Daily, J. Distel, K. Lande, C.K. Lee et al., in Proceedings of the 23rd International Cosmic Ray Conference (University of Calgary, Alberta, Canada, 1993), Vol. 3, p. 865.

[15] J. Engel, S. Pittel, and P. Vogel, Phys. Rev. C50, 1702 (1994).

[16] G. K. Schenter and P. Vogel, Nucl. Sci. \& Eng. 83, 393, (1983).

[17] E. Kolbe has made this replacement, though without shortening the wavelength, in several papers.

[18] J. Engel, E. Kolbe, K. Langanke, and P. Vogel, Phys. Rev. C54, 2740 (1996).

[19] T.K. Gaisser, T. Stanev, and G. Barr, Phys. Rev. D38, 85 (1988); G. Barr, T.K. Gaisser, and Todor Stanev, Phys. Rev. D39, 3532 (1989). 